\title{
METTL3-mediated m6A modification of KIF3C-mRNA promotes prostate cancer progression and is negatively regulated by miR-320d
}

\author{
Honggui Ma' ${ }^{1}$, Facai Zhang ${ }^{2}$, Quliang Zhong ${ }^{2}$, Jianquan Hou ${ }^{1}$ \\ ${ }^{1}$ The Department of Urology, The First Affiliated Hospital of Soochow University, Suzhou 215031, Jiangsu, China \\ ${ }^{2}$ The Department of Urology, The Affiliated Hospital of Guizhou Medical University, Guiyang 550009, Guizhou, \\ China
}

Correspondence to: Jianquan Hou; email: hou iq@126.com, https://orcid.org/0000-0003-2257-936X Keywords: METTL3, m6A, KIF3C, miR-320d, prostate cancer

Received: March 3, $2021 \quad$ Accepted: August 14, 2021

Published: September 19, 2021

Copyright: (C) $2021 \mathrm{Ma}$ et al. This is an open access article distributed under the terms of the Creative Commons Attribution License (CC BY 3.0), which permits unrestricted use, distribution, and reproduction in any medium, provided the original author and source are credited.

\section{ABSTRACT}

The occurrence of distant metastasis is one of the leading causes of death in patients with prostate cancer (PCa). It is confirmed that kinesin protein is associated with a variety of malignancies, and the KIF3 family is related to cancer, but the relationship between KIF3C and prostate cancer is not clear. Our experiments have confirmed that KIF3C is highly expressed in prostate cancer tissues and cell lines. Further, functional tests have proven that KIF3C can promote the growth migration and invasion of PCa. We used Starbase 3.0 to discover that methyltransferase like 3 (METTL3) interacts with KIF3C. Our hypothesis and experiments concluded that METTL3 induced m6A modification on KIF3C, promoting the stabilization of KIF3C-mRNA by IGF2 binding protein 1 (IGF2BP1). The prediction that miR-320d inhibits KIF3C expression by targeting METTL3 using the miRmap website, was later confirmed experimentally. Further, a recovery experiment was used to confirm that miR-320d inhibited the progression of prostate cancer. KIF3C was overexpressed in prostate cancer, promoting its growth migration and invasion was induced by miR-320d/METTL3 in an m6A dependent process.

\section{INTRODUCTION}

Prostate cancer $(\mathrm{PCa})$ is currently one of the most common malignancy found across the world, and the second main cause of cancer-related deaths in males [1]. Although, the popularity of prostate-specific antigen (PSA) screening technology has increased the rate of diagnosis and the treatment of prostate cancer, where early prostate cancer can be completely cured, yet about $10 \%$ to $20 \%$ of the newly diagnosed prostate cancer patients are at their advanced stages [2]. These patients are usually given androgen deprivation therapy (ADT). However, hormone therapy can only last for a median of two years without progression [3]. Therefore, understanding the mechanism of prostate cancer progress is of great significance in treating the disease.
Kinesin superfamily proteins (KIFs) were discovered in 1985 [4]. They are found in eukaryotic cells, and more than 650 members have been discovered till date. KIFs are a class of proteins that move unidirectionally along the microtubules and participate in a variety of intracellular physiological activities, including the transport of organelles and protein molecules, in cell morphology and cytoskeleton dynamics $[5,6]$. KIF3 is one of the subfamilies of the KIFs, which includes three members: KIF3A, KIF3B, and KIF3C, and they all are reported to be associated with malignancy. KIF3C is highly expressed in the nervous system and is involved in anterograde axonal transport in mouse neuronal cells [7]. A recent study revealed that KIF3C is an injury specific kinesin that contributes to axon growth and regeneration by regulating and organizing the microtubule cytoskeleton in the growth cone [8-12]. In 
the tumor diseases, accumulated researches indicated that KIF3C exerted as an oncogene involving in glioma, breast cancer progress by activating $\mathrm{PI} 3 \mathrm{~K} / \mathrm{AKT} / \mathrm{mTOR}$ and TGF- $\beta$ signaling pathway $[13,14]$. However, these studies have focused on studying KIF3C in normal mouse cells and other tumor disease, but its research on human prostate cancer needs in-depth studies.

N6-methyl adenosine (m6A) is the most common modification method in mRNA $[15,16]$. It adds a methyl group to the mRNA, and then demethylase, catalyzed by N6-methyltransferase removes the methyl group, dynamically regulating the mRNA modification, which affects biological processes such as RNA stability, nucleation, splicing or translation [15]. Studies have shown that m6A RNA modification plays an important role in the pathogenesis of human diseases, and any abnormality in this modification may cause tumorigenesis [17, 18]. Methyltransferase like 3 (METTL3) is a key catalyst in m6A modification and plays a carcinogenic role in a variety of cancers, such as stomach cancer [19], bladder cancer [20], and pancreatic cancer [21]. However, the relationship between METTL3 and prostate cancer is unclear. Additionally, recent evidence suggested that genetic variation in miRNA and its targets may be related to the efficacy of androgen deprivation therapy (ADT) in patients with prostate cancer [22]. Hence, we aimed to explore the relationship between METTL3, KIF3C, and miR-320d.

In our study, we found that the KIF3C level is increased in prostate cancer tissues, and down-regulation of KIF3C can inhibit the proliferation and invasion of prostate cancer cells. Bioinformatics predicted a regulatory relationship between KIF3C and METTL3mediated m6A modification. Hence, our study aimed to explore the role of the regulatory mechanism of KIF3C, METTL3, and miR-320d in developing prostate cancer.

\section{RESULTS}

KIF3C is overexpressed in PCa and is negatively correlated with the prognosis

Firstly, the expression of KIF3C in PCa was explored to figure out its participation in the disease. The immunohistochemistry results showed that the expression of KIF3C was higher in prostate cancer tissues than in the adjacent tissues (Figure 1A). While KIF3C expression was detected in tumor and adjacent normal tissues as well (Figure 1B). The clinical relevance of KIF3C was built on an excel sheet (Table 1), the results indicated that KIF3C is positively with $\mathrm{PCa}$ lymph node metastasis and Seminal vesicle invasion.

\section{KIF3C silencing prevented proliferation, migration and invasion of PCa cells}

Secondly, the biological function of KIF3C was explored. The KIF3C expression in prostate cancer cell lines was detected by q-RT-PCR and western blotting, the results indicated that KIF3C was overexpressed in the prostate cell lines. (Figure 2A, 2B). KIF3C was silenced in $\mathrm{C} 4-2 \mathrm{~B}$ and DU145 cells, which expressed the highest KIF3C level (Figure 2C, 2D). Silencing KIF3C reduced growth, migration and invasion of both the $\mathrm{PCa}$ cell lines (Figure 2E-2G). Overall, knocking down $\mathrm{KIF} 3 \mathrm{C}$ prevented the $\mathrm{PCa}$ proliferation, migration and invasion in vitro.

\section{METTL3 facilitated IGF2BP1-regulated stabilization of KIF3C mRNA}

Thirdly, the mechanism of KIF3C upregulation in PCa was interrogated. Using Starbase 3.0 (http://starbase. sysu.edu.cn/), we found that METTL3 can interact with KIF3C. Recent studies have found
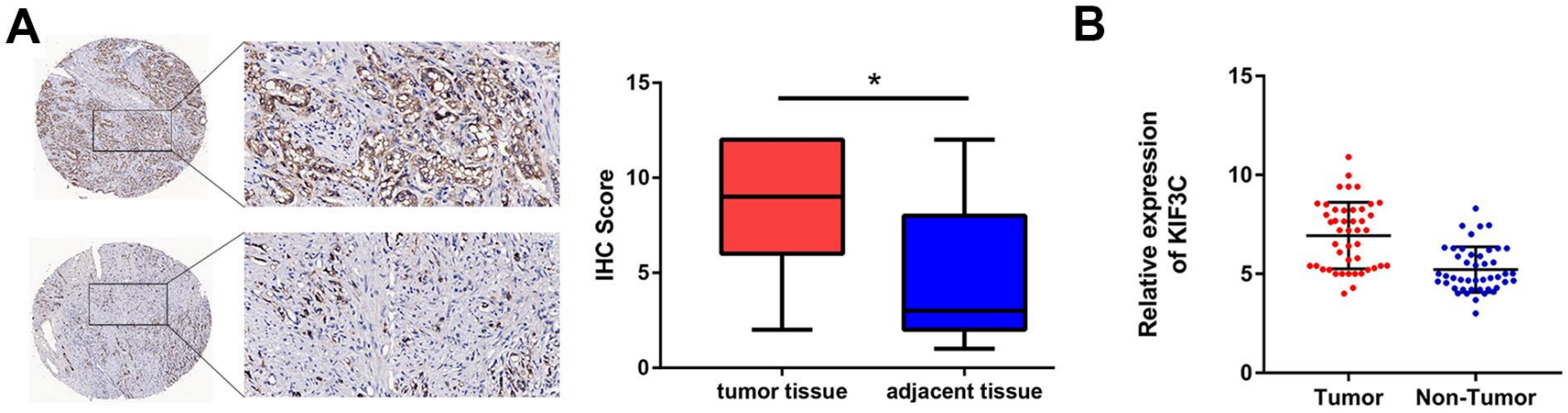

Figure 1. KIF3C is overexpressed in PCa. (A) Immunohistochemistry of tumor and adjacent tissues was conducted to show KIF3C levels (80 cases). (B) RT-qPCR showing KIF3C expression in tumor and adjacent tissues. Data are reported as means \pm standard deviation of three independent experiments. $* p<0.05 ; * p<0.01$. 
Table 1. The correlation of KIF3C expression with the prostate cancer clinical features.

\begin{tabular}{|c|c|c|c|c|c|}
\hline \multirow{2}{*}{ Variable } & \multirow{2}{*}{ Group } & \multicolumn{3}{|c|}{ KIF3C protein expression } & \multirow{2}{*}{$P$ value } \\
\hline & & $\mathbf{n}$ & High & Low & \\
\hline \multirow{2}{*}{ Age } & $<70$ & 45 & 28 & 17 & \multirow{2}{*}{0.474} \\
\hline & $\geq 70$ & 35 & 19 & 16 & \\
\hline \multirow{2}{*}{ Lymph node metastasis } & Positive & 17 & 14 & 3 & \multirow{2}{*}{0.026} \\
\hline & Negative & 63 & 33 & 30 & \\
\hline \multirow{2}{*}{ Surgical margin status } & Positive & 14 & 10 & 4 & \multirow{2}{*}{0.289} \\
\hline & Negative & 66 & 37 & 29 & \\
\hline \multirow{2}{*}{ Seminal vesicle invasion } & Positive & 26 & 20 & 6 & \multirow{2}{*}{0.022} \\
\hline & Negative & 54 & 27 & 27 & \\
\hline \multirow{2}{*}{ PCa stage } & $\mathrm{T} 1$ & 53 & 29 & 26 & \multirow{2}{*}{0.105} \\
\hline & $\mathrm{T} 2 / \mathrm{T} 3$ & 27 & 18 & 7 & \\
\hline \multirow{3}{*}{ Preoperative PSA } & $<4$ & 2 & 1 & 1 & \multirow{3}{*}{0.038} \\
\hline & $4-10$ & 33 & 14 & 19 & \\
\hline & $>10$ & 45 & 32 & 13 & \\
\hline \multirow{3}{*}{ Gleason score } & $<7$ & 31 & 15 & 16 & \multirow{3}{*}{0.035} \\
\hline & 7 & 27 & 14 & 13 & \\
\hline & $>7$ & 22 & 18 & 4 & \\
\hline \multirow{2}{*}{ Angiolymphatic invasion } & Positive & 20 & 15 & 5 & \multirow{2}{*}{0.6576} \\
\hline & Negative & 60 & 32 & 28 & \\
\hline \multirow{2}{*}{ Biochemical recurrence } & Absence & 59 & 34 & 25 & \multirow{2}{*}{0.7324} \\
\hline & Presence & 21 & 13 & 8 & \\
\hline
\end{tabular}

that METTL3 can catalyze m6A modification of mRNA, thereby upregulating the related gene expression and promoting cancer progression [23]. Therefore, we hypothesized that METTL3 can induce KIF3C expression. The expression of METTL3 in prostate cancer cell lines determined that it is highly expressed in prostate cancer (Figure 3A and Supplementary Figure 1A). The knockout of METTL3 was confirmed in PCa cell lines though RT-PCR (Figure 3B and Supplementary Figure 1B). RIP assay detected a large amount of KIF3C-mRNA in the METTL3 antibody precipitate (Figure 3C). Also, the m6A RIP assay illustrated that silencing METTL3 reduced the m6A modification in KIF3C-mRNA (Figure 3D). The m6A modification could reportedly strengthen mRNA stability. Hence, we examined the effect of METTL3 on the stability of KIF3C-mRNA. RT-qPCR results, after the addition of actinomycin D, showed that METTL3 silencing disrupted the mRNA stability of KIF3C (Figure 3E). Further, through RMBase 2.0 (http://rna.sysu.edu.cn/rmbase/), we found a binding site for m6A in KIF3C and predicted that IGF2BP1 can interact with KIF3C (Supplementary Figure 2). Studies have shown that m6A modification promotes the binding of IGF2BP1 to the target mRNA, thereby promoting the stability of
mRNA [24]. Therefore, we tested whether METTL3 can stabilize KIF3C-mRNA by IGF2BP1 or not. RIP assay confirmed that IGF2BP1 interacts with KIF3CmRNA, and METTL3 silencing reduces KIF3CmRNA enrichment in IGF2BP1 precipitation (Figure 3F). The half-life of KIF3C-mRNA was reduced after down-regulating IGF2BP1 (Figure 3G). Also, silencing METTL3 or IGF2BP1 reduced KIF3CmRNA and protein expression in $\mathrm{PCa}$ cells (Figure 3H, 3I).

\section{METTL3 is the target gene of miR-320d}

The upstream mechanism of METTL3/KIF3C in PCa is discussed further. It is believed that miRNAs are regulators of gene expression, and they participate in cancer progression [25]. The miRmap (https://mirmap. ezlab.org/) was explored to find potential miRNAs that target METTL3. The miR-320d interacted effectively with METTL3 and was chosen for further research. The low expression of miR-320d in PCa cell lines and tissues were confirmed by qPCR (Figure 4A, 4B). The binding sequences on METTL3 for miR-320d and the mutated sites are shown in Figure 4C. Overexpression of miR320d significantly reduced the luciferase activity of METTL3 WT but did not reduce the luciferase activity of 
METTL3 Mut (Figure 4D). RIP assay identified coimmunoprecipitation of miR-320d and METTL3 in two PCa cell lines by the Ago 2 antibody (Figure 4E). Next, miR-320d was also overexpressed in two PCa cell lines using miR-320d mimic (Figure 4F). Overexpression of miR-320d down-regulated the mRNA and protein expression of METTL3 (Figure 4G, 4H). Furthermore, the m6A level of $\mathrm{PCa}$ cells and tissue were higher than normal prostate tissue and cells (Figure 4I, 4J). Also, the expression of miR-320d was negatively correlated with m6A level in PCa tissues. (Figure 4K).

\section{miR-320d inhibited $P C$ progression through METTL3/KIF3C}

Finally, we confirmed if miR-320d can regulate $\mathrm{PCa}$ progress by recovery experiment. The expression of KIF3C was lowered in the case of overexpression of miR-320d, and restored by overexpression of METTL3 or KIF3C (Figure 5A). The proliferation, invasion, and migration of $\mathrm{PCa}$ were retarded by miR-320d mimic and rescued by the ectopic expression of METTL3 or KIF3C (Figure 5B-5D).

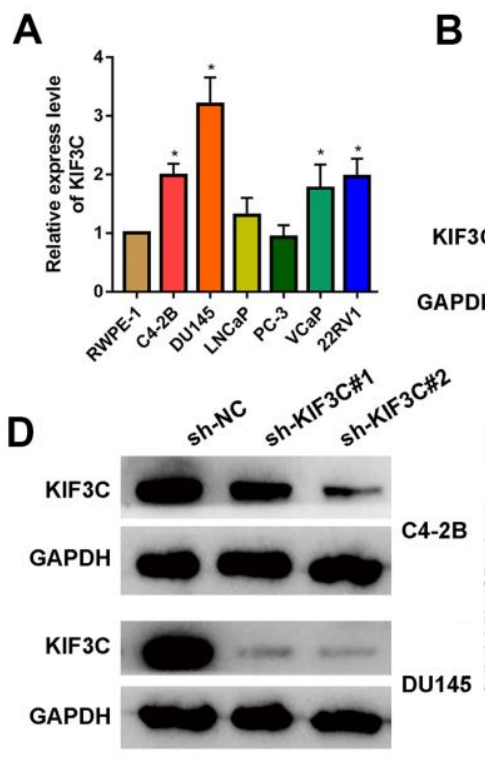

C
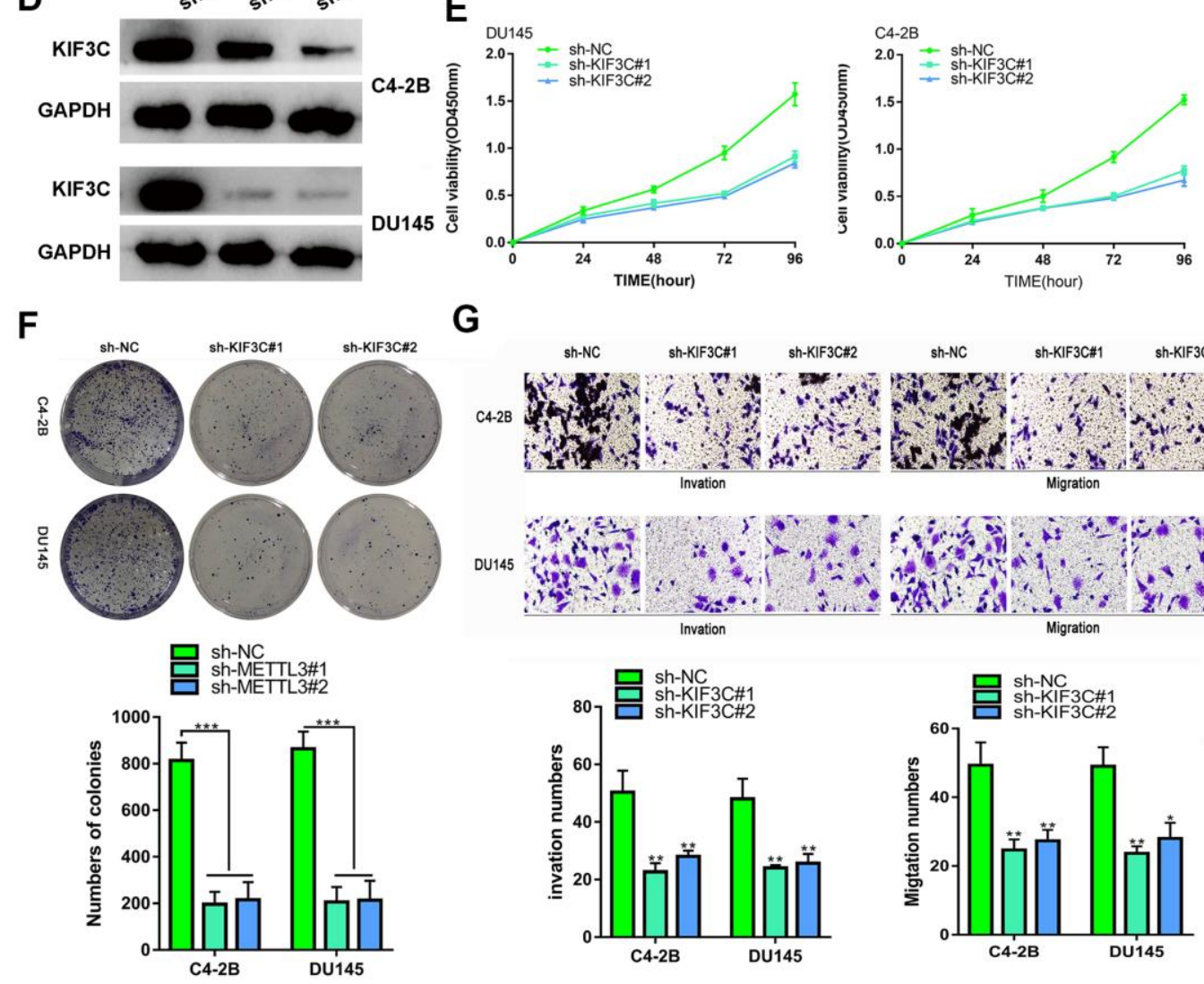

G
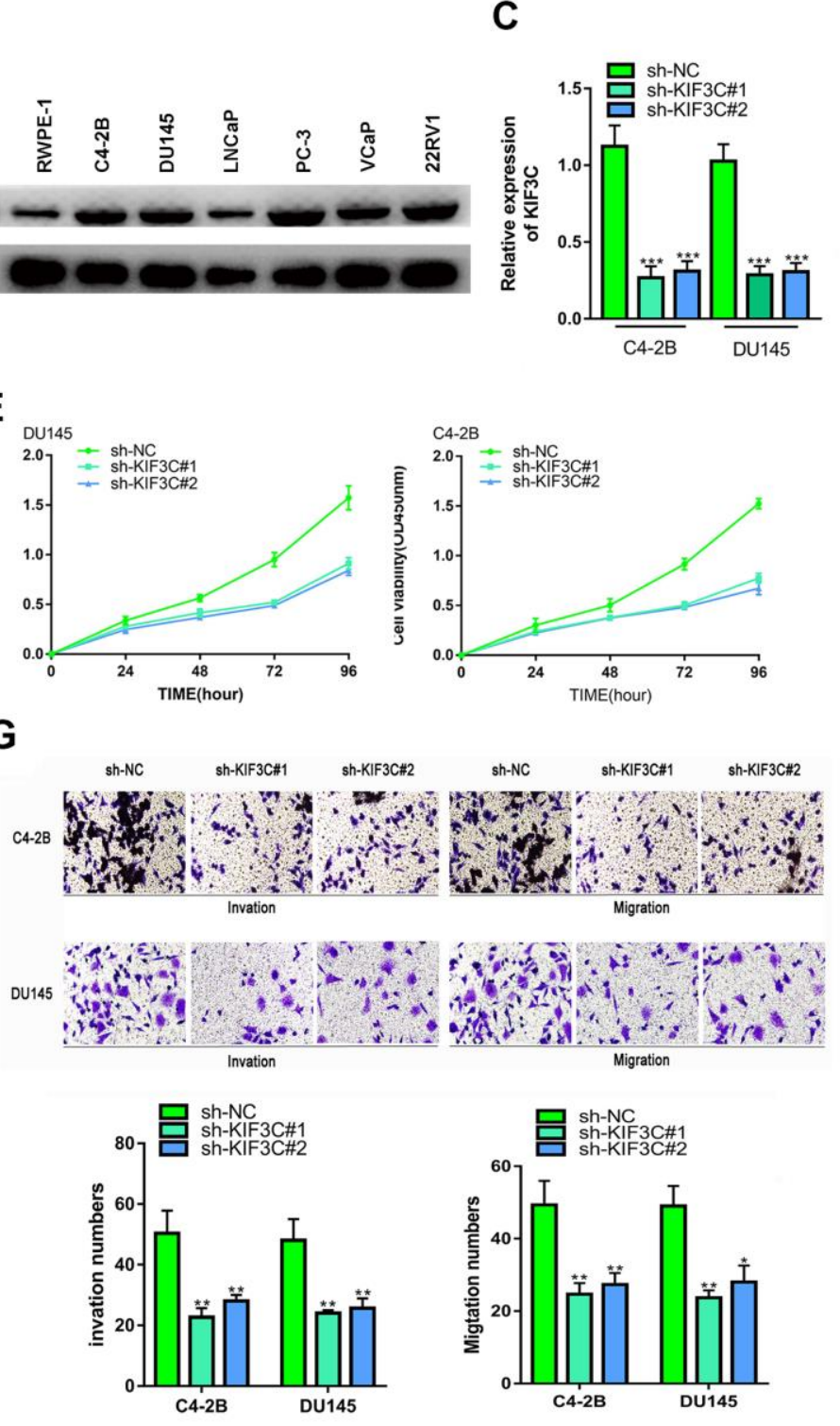

Figure 2. KIF3C silence prevented the proliferation migration and invasion of PC cells. (A, B) RT-qPCR and western blotting showing KIF3C expression in PCa cell lines. (C, D) RT qPCR and western blotting showing KIF3C knockdown expression in C4-2B and DU145 cells. (E, F) CCK8 and cloning formation assays showing proliferation after KIF3C knockdown in PCa cells. (G) Transwell showing invasiveness and migration capability of PCa cells after knocking down KIF3C. Data are reported as means \pm standard deviation of three independent experiments. ${ }^{*} p<0.05 ;{ }^{*} p<0.01$. 

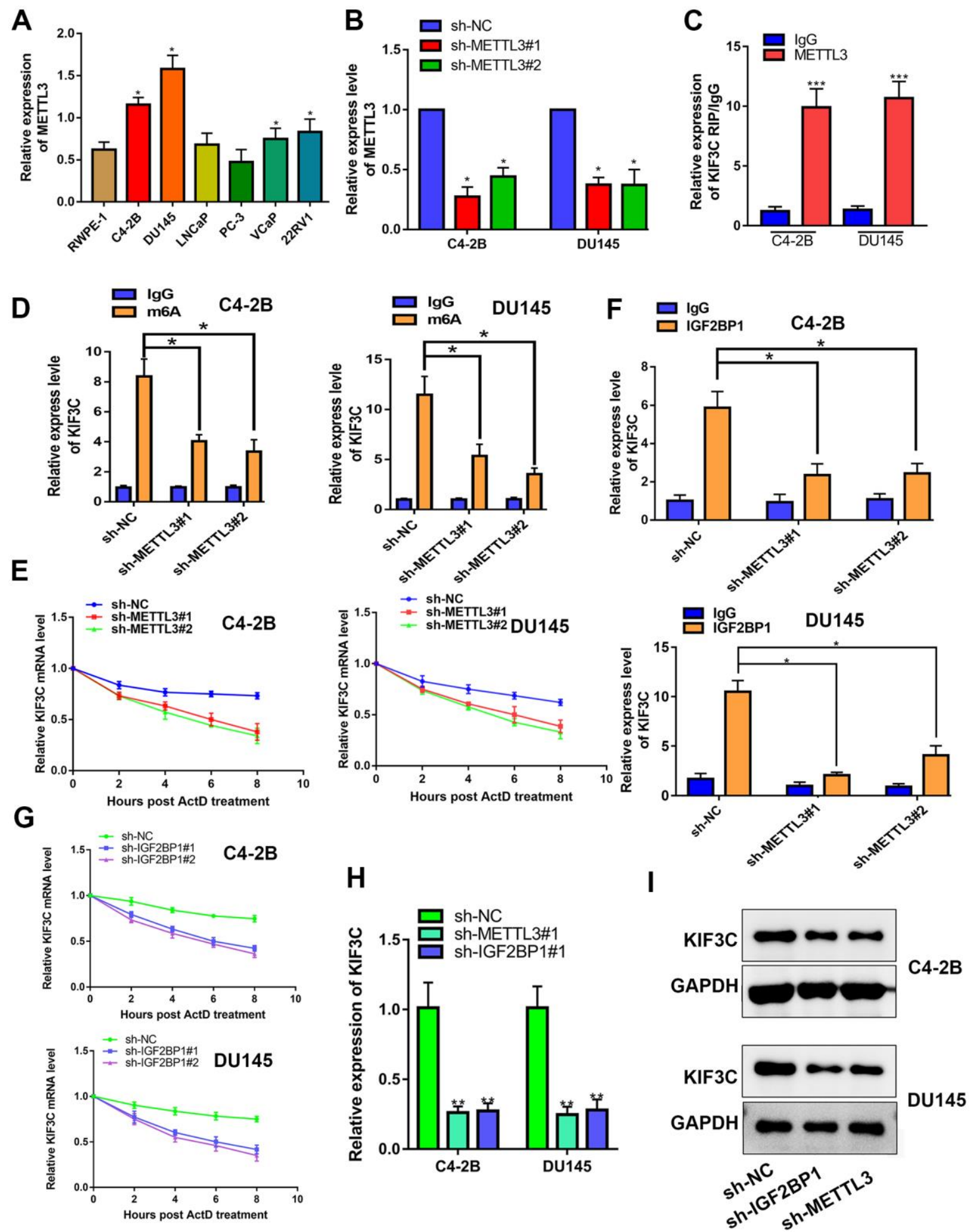

Figure 3. METTL3 facilitated IGF2BP1-regulated stabilization of KIF3C mRNA. (A) RT-qPCR showing METTL3 expression in PCa cell lines. (B) RT-qPCR showing METTL3 knockdown expression in C4-2B and DU145 cells. (C) RIP assay was conducted to show the interaction between METTL3 and KIF3C in PCa cells. (D) m6A RIP assay was conducted to show the m6A modification on KIF3C mRNA in C4-2B and DU145 cells. (E) RT qPCR was conducted to show the stability of KIF3C mRNA after adding ActD. (F) RIP assay was conducted to show the effects of the down-regulation of METTL3 on the interaction between IGF2BP1 and KIF3C in C4-2B and DU145 cells. (G) RT-qPCR showing the stability of KIF3C mRNA after adding ActD in C4-2B and DU145 cells. (H, I) RT-qPCR and western blot were conducted to confirm the KIF3C expression under METTL3 or IGF2BP1 silencing. Data are reported as means \pm standard deviation of three independent experiments. ${ }^{*} p<$ $0.05 ; * *<0.01$. 


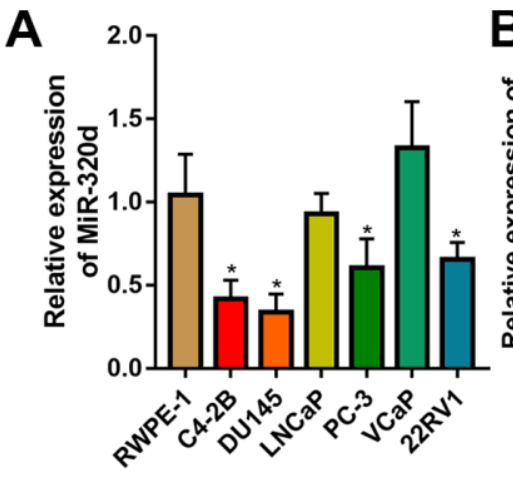

B

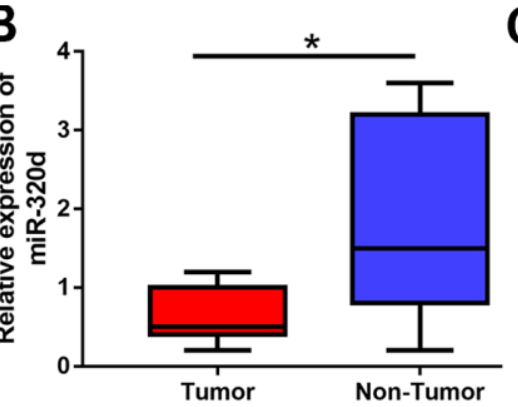

C

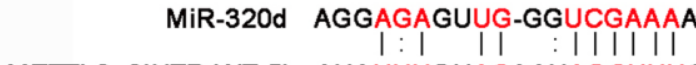

METTL3 3'UTR WT 5' AUAUUUGUACAAUAGCUUUC METTL3 3'UTR MUT 5' AUATTTGUACAAUAGCTTTC

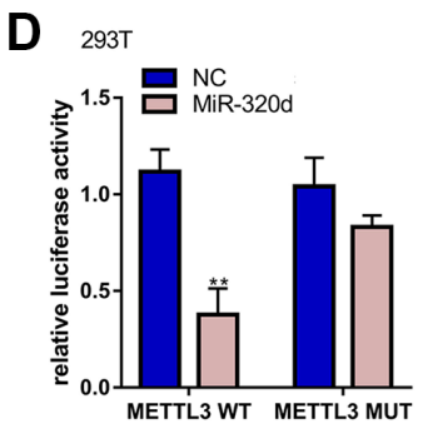

E

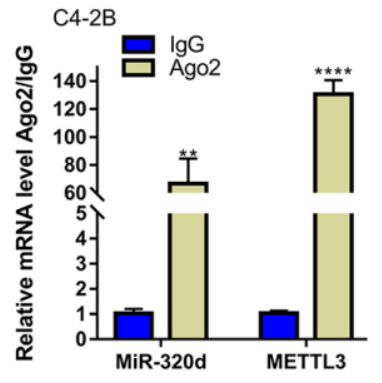

$\mathbf{F}$

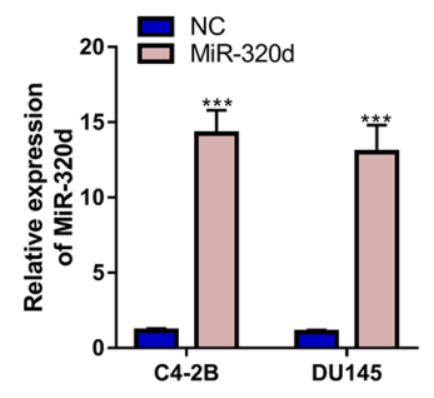

G

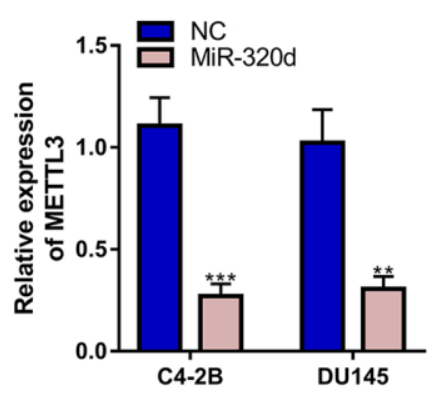

H

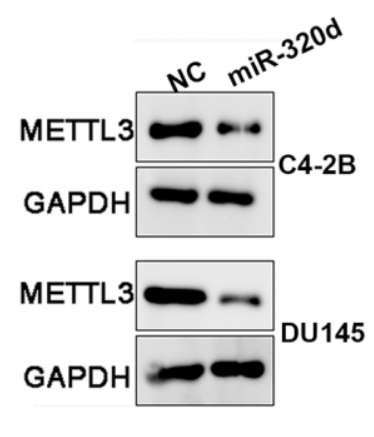

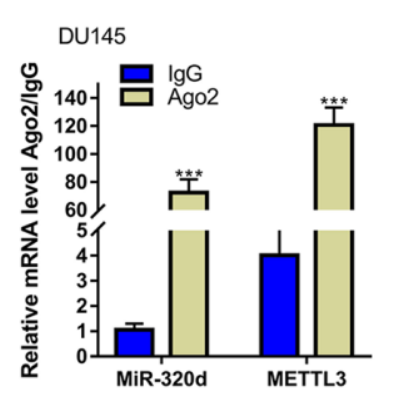

. 
KIF3C silence prevented the proliferation of PC cells in vivo

To further evaluate the effects of KIF3C on tumorigenesis in vivo, we conducted a xenograft tumor model by subcutaneously injecting the C4-2B PCa cells.
The representative xenograft tumors from each group at 5 weeks were photographed (Figure 6A). The weigh and volume of the implanted tumors showed that KIF3C knockdown PCa cells exerted more slower growth ability (Figure 6B, 6C). Immunohistochemistry (IHC) staining analysis of the correlation of KIF3C expression

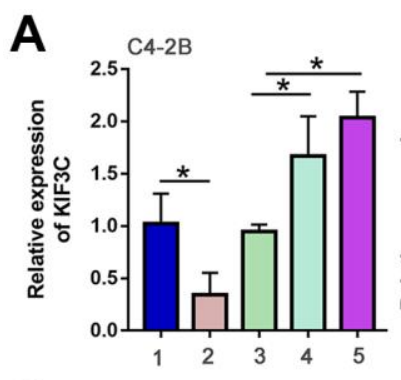

C

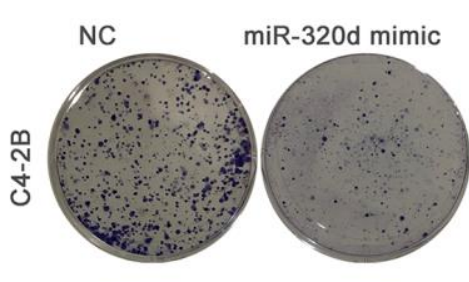

miR-320d mimic
B
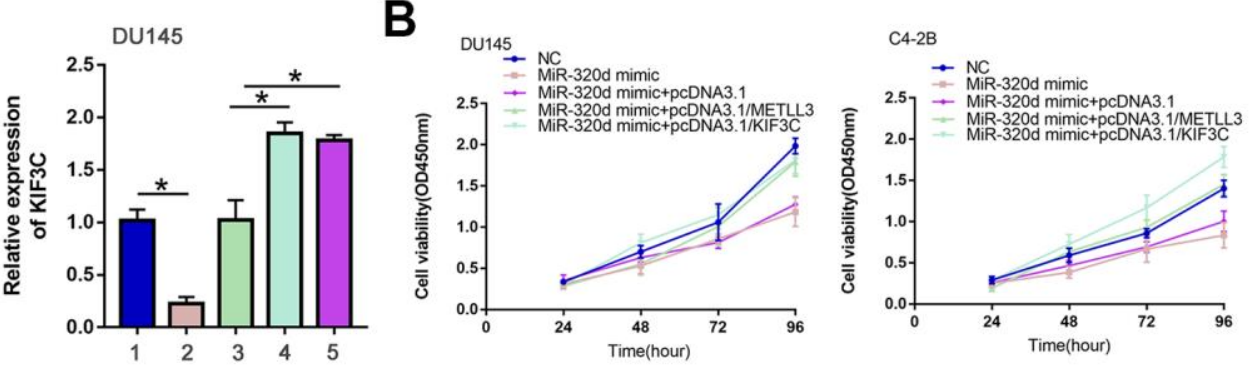

+pcDNA3.1 +pcDNA3.1/METLL3 +pcDNA3.1/KIF3C
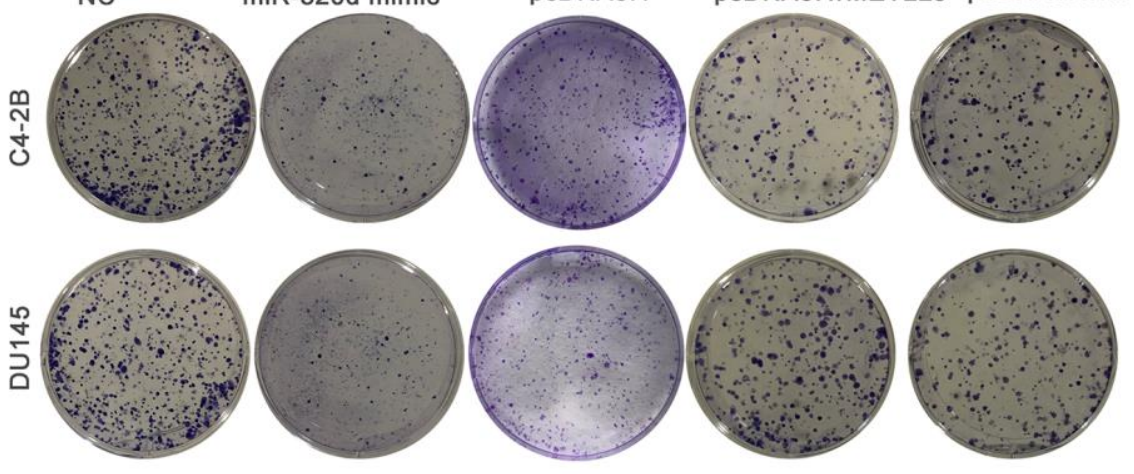
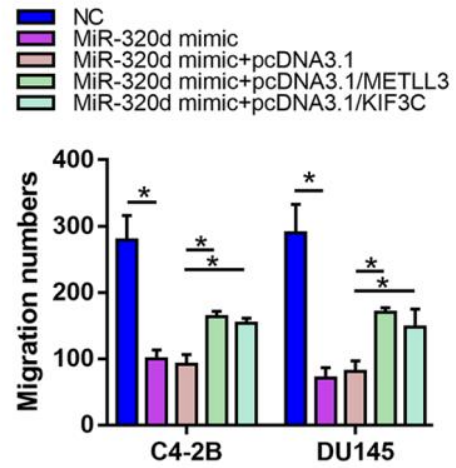

D
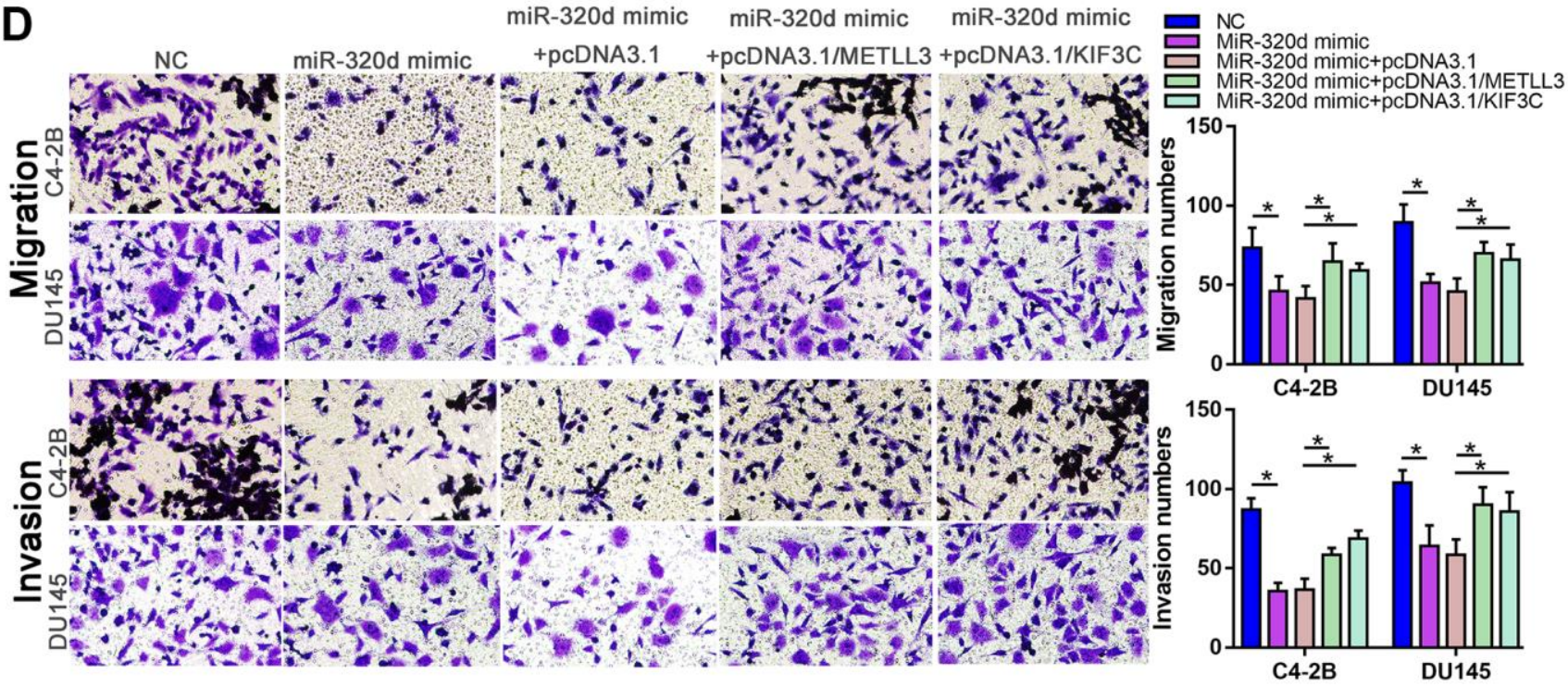

Figure 5. MiR-320d inhibited PC progression through METTL3/KIF3C. C4-2B and DU145 cells were transfected with NC mimic, miR320d mimic, miR-320d mimictpcDNA3.1, miR-320d mimic+pcDNA3.1/METTL3, or miR-320d mimic + pcDNA3.1/KIF3C, respectively. (A) RTqPCR showing KIF3C expression in indicated group PCa cells. (B, C) CCK-8 and colony formation were conducted to show the proliferation of PCa cells. (D) Transwell showing invasion and migration ability of PCa cells. Data are reported as means \pm standard deviation of three independent experiments. ${ }^{*} p<0.05 ; * * 0.01$. 
of the KI-67, PCNA and the two markers were significantly downregulated in the KIF3C silenced groups (Figure 6D).

\section{DISCUSSION}

The occurrence and development of prostate cancer are closely related to the abnormal expression of oncogenes [26]. Earlier studies have found that the kinesin family's proliferation, invasion, and migration are closely related to tumors [27]. KIF3C, a newly discovered prostate cancer molecule in our study, also exists in other cancers $[14,28]$. KIF3C is reported to express highly in breast cancer and promote tumor progression [14]. Similarly, we found that KIF3C is highly expressed in prostate cancer and is closely related to the prognosis of the disease as well. Functional tests have also shown that it can promote proliferation, invasion, and migration, but the reason behind its high expression and the molecular mechanism of promoting tumorigenesis and development remains unclear.

In eukaryotes, the 5'-cap and 3'-polyA modifications play a very important role in regulating transcription, and the internal modifications of mRNA help in maintaining its stability [29]. The most common internal modifications of mRNA include N6methyladenosine (m6A), N1- methyladenosine (m1A), 5- methylcytosine (m5C), and so on. The m6A modification can accelerate the processing time of the mRNA precursors, speed of mRNA transport, and nucleation in cells $[30,31]$. Therefore, we used bioinformation based predictions to find the presence of m6A modification in KIF3C and hypothesized that $\mathrm{KIF} 3 \mathrm{C}$ overexpression in prostate cancer is mediated by its m6A modification. Later, RIP assays explored that m6A can bind to KIF3C and regulate its expression. The m6A methylase METLL3 has been studied in a variety of tumors and is closely related to the tumor's high invasive capacity and rapid appreciation $[32,33]$. The expression of m6A and KIF3C decreased after knocking down METLL3. This conclusion is consistent with the previous assumptions. Further, through RIP assay and bioinformatics prediction, we found that IGF2BP1 can be used as a methylation recognition enzyme for m6A modification of KIF3C. The IGF2BP1 family is reported in the literature, as an m6A methylase and promoter of cancer [24]. Thus, we tested the expression and stability of KIF3C after knocking down IGF2BP2. The results showed that the expression and
A

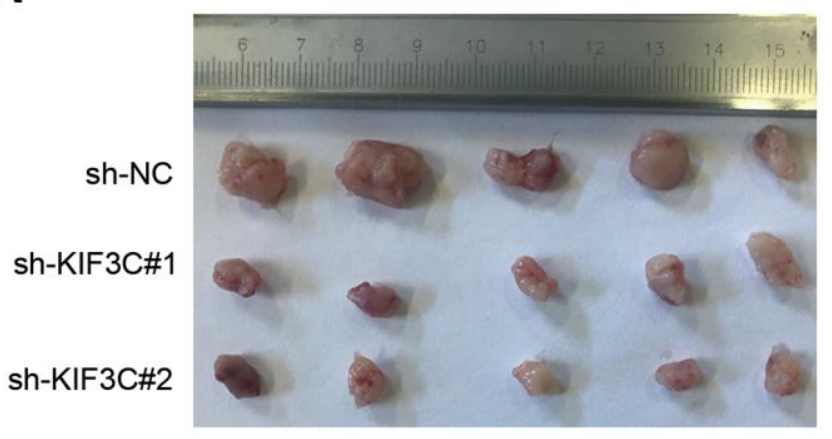

C

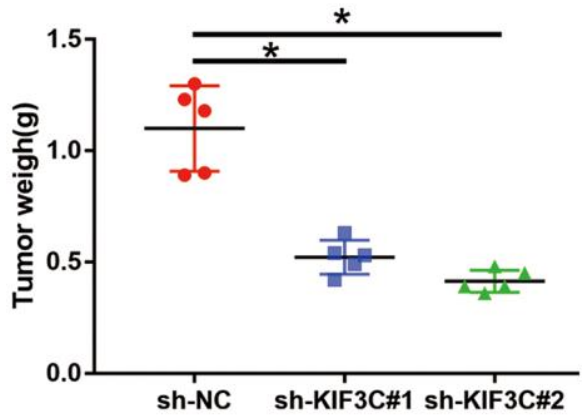

B

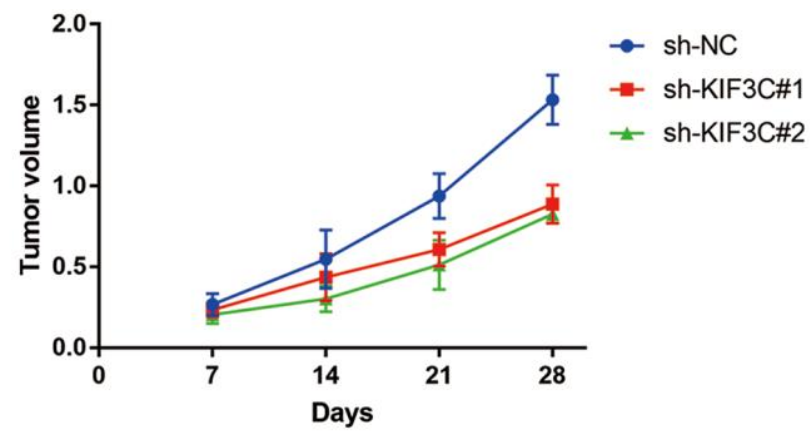

D

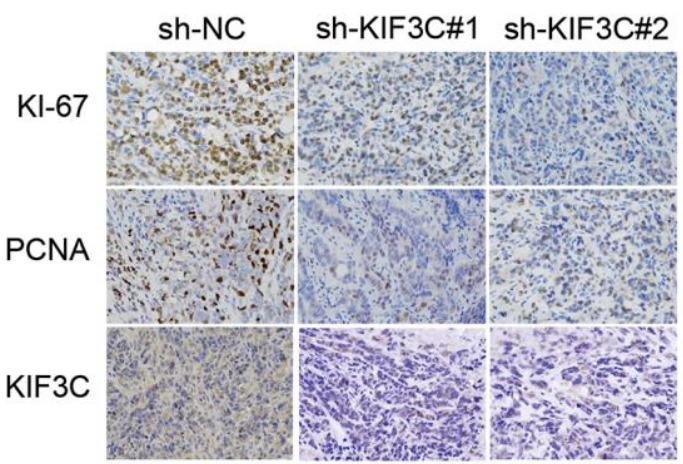

Figure 6. KIF3C silence prevented the proliferation of PC cells in vivo. (A) Represent image of nude mouse tumors ( $n=5$ ). (B) The implanted tumor volume of the KIF3C knockdown PCa cells nude mouse. (C) The implanted tumor weight of the KIF3C knockdown PCa cells nude mouse. (D) Typical IHC staining images showing Ki-67, PCNA and KIF3C of the implanted tumors. Data are reported as means \pm standard deviation of three independent experiments. ${ }^{*} p<0.05 ; * * p<0.01$. 
stability of KIF3C are regulated by IGFBP1, and this process is m6A-dependent.

The role of miRNA in cancer is widespread [34]. It is unclear whether miRNAs can participate in the regulation of m6A or not. We found that METTL3 can be regulated by miRNA-320d through bioinformatics prediction and further studied the regulatory relationship between miR-320d and METTL3. Expression and dual fluorescence experiments showed that miR-320D can target METTL3 and regulate its expression. Further confirmation by molecular and cellular function tests showed that miRNAs can inhibit $\mathrm{PCa}$ progression through METTL3 and KIF3C.

In conclusion, KIF3C was overexpressed in the prostate cancer and exerted as an oncogene involving in promoting proliferation and metastasis, which was regulated by METTL3 in m6A modification dependence.

\section{MATERIALS AND METHODS}

\section{Tissue specimen}

The Specimens and the clinical-pathological data collection were approved by the Institutional Research Ethics Committee of the affiliated hospital of Guizhou Medical University. The IHC analysis of prostate cancer was provided by the affiliated hospital of Guizhou Medical University. The correlation between KIF3C and prostate cancer patients' clinical features was analyzed and confirmed before specimen collection. A total of 80 patients' prostate cancer tissues and adjacent tissues were collected between 2015 to 2019 from the urology department of the affiliated hospital of Guizhou Medical University for immunohistochemical detection. All the patients' diagnostic criteria were classified as prostate cancer, according to the World Health Organization's diagnostic criteria. The Specimen and clinical pathological data collection were approved by the Institutional Research Ethics Committee of the affiliated hospital of Guizhou Medical University (approval no. GMU2019-058). All patients provided written informed consent prior to enrollment in the study.

\section{Cell culture}

The human PCa cell lines, C4-2B (prostate cancer), DU145 (prostate cancer brain metastases), LNCaP (prostate cancer Lymph node metastasis), PC-3 (prostate cancer bone metastases), RWPE1 (prostate cancer), 22RV1 (prostate cancer), VCaP (vertebral metastasis) were acquired from the Shanghai Chinese Academy of Sciences cell bank (China). All the cells were cultured in high glucose DMEM medium
(Hyclone) with $10 \%$ fetal bovine serum (Gibco) and grown in a cell culture incubator with $5 \% \mathrm{CO}_{2}$ at $37^{\circ} \mathrm{C}$.

\section{Cell transfection}

The cells were plated on to a six-well plate and grown until the confluency reached 50\%. For transfection, siRNA, and Lipofectamine 2000 were diluted in serumfree medium, mixed and kept separately for a few minutes. Next, the transfection reagent and the siRNA diluent were mixed by pipetting 3-5 times gently and allowed to stand for a few minutes at room temperature. This mix was then added to the six-well plate, shaken, and incubated at $37^{\circ} \mathrm{C}$ with $5 \% \mathrm{CO}_{2}$ for $24-48 \mathrm{~h}$. Fresh medium was added after 4-6 h of transfection. According to the instruction, the concentration of the miRNA or siRNA is $0.05 \mathrm{nmol} / \mathrm{ml}$ and the transfection $48 \mathrm{~h}$ for RNA and protein extracting. The sequences of short hairpin RNA (shRNA) interference are as follows: sh-KIF3C\#1: 5'-CGAACCGAGCCAAGAACAUUC UCUUGAAAU GUUCUUGGC UCGGUUCGGGG-3', sh-KIF3C\#2: 5'GCUGCCCAAGACCUUCACUUCUCUUG AAAGUG AAGGUCUUGGGCAGCGGG-3', a scramble sequence was used for negative control (NC). SiRNA of METTL3: CTGCAAGTATGTTCACTATGA, siRNA of IGF2BP1: ACGCTTAGAGATTGAACATTC. And these shRNA or siRNA were purchased from Ribobio (Guangzhou, China).

\section{Quantitative real-time polymerase chain reaction (RT-qPCR)}

RNA from each group was extracted using Trizol Reagent (Invitrogen, CA, USA) according to the instructions. Then, cDNA was synthesized using the PrimeScript RT reagent kit (Takara) according to the manufacturer's protocol. Real-time quantitative PCR was performed using the Powerup SYBR Green PCR Master Mix (Life Technologies).

\section{Cell viability analysis}

Cell Counting Kit-8 (Beyotime, China) was used to detect cell proliferation capacity. As per the instructions, $100 \mathrm{uL}$ of $2 \times 10^{3}$ cells were seeded into a 96-well plate, and $10 \mu \mathrm{L}$ of CCK8 solution was added to each well. Absorbance values were measured at different time points of $0,24,48,72$, and $96 \mathrm{~h}$.

\section{Colony formation assay}

Each group of cells, in their exponential growth phase, were seeded into a six-well plate at 1000 cells per well, and then $2 \mathrm{~mL}$ of 10\% FBS supplemented DMEM medium was added to each well, mixed and incubated for 7 to 14 days. Later, it was fixed with $4 \%$ paraformaldehyde and stained with $0.5 \%$ crystal violet. 


\section{Transwell assay}

Corning's 24-well Transwell system was used for the invasion and transfer experiments. $1 \times 10^{5}$ cells were mixed with $200 \mu \mathrm{L}$ of serum-free medium and added to each of the upper chambers. Subsequently, $700 \mu \mathrm{L}$ of $10 \%$ FBS medium was added to the lower chamber. After $24-48 \mathrm{~h}$ of incubation, the upper chamber was removed and stained with $0.2 \%$ crystal violet. The migrated cells in the lower chamber were photographed and counted.

\section{Western blot analysis}

Cells from each group were lysed using RIPA (Beyotime, China) to extract the protein. After quantifying the protein using BCA reagents (Beyotime, China), the sample was re-diluted to the same concentration, boiled, and stored in a refrigerator at $20^{\circ} \mathrm{C}$ until further use. SDS-PAGE gel with $10 \%$ separation gel and 5\% concentrated gel was prepared using Solarbio's (China) reagents, according to the manufacturer's procedure. Samples were electrophoresed, transferred, and then finally exposed. The protein content we add each time is $30 \mu \mathrm{g}$.

\section{Reporter gene transfection and luciferase activity assay}

The cells were seeded in a six-well plate, and after reaching $80 \%-90 \%$ confluency, they were cotransfected with a luciferase reporter gene, miR-320d, or METTL3 containing TA promoter, for $4-6 \mathrm{~h}$ using LipofectAMINE reagent (Invitrogen). After $24 \mathrm{~h}$ of transfection, the luciferase activity in the cell extract was measured using a double luciferase gene detection kit (Beyotime, China).

\section{RNA immunoprecipitation (RIP) assay}

The Magna RIPTM Quad RNA-Binding Protein Immunoprecipitation Kit (Millipore, MA, USA) was used to analyze Protein-RNA Interaction. The total RNA was mixed with antibodies (against $\mathrm{IgG}, \mathrm{m} 6 \mathrm{~A}$, IGF2BP1, or Ago2) or rabbit IgG-binding protein A/G magnetic beads in 1x IP buffer. The RNA of interest was immunoprecipitated with the beads. All the antibodies were provided by Magna.

\section{IHC analysis}

The expression of KIF3C was detected by immunohistochemical staining. The tissue was cut into 3um slice and then dewaxed. Next, these sections were incubated with rabbit anti-KIF3C monoclonal (1: 200; 14333-1-AP; Proteintech, IL, USA) antibody at $4^{\circ} \mathrm{C}$ overnight. After washing three times with PBS, each section was incubated with goat anti-rabbit IgG for 30 min and then developed using 3,3' diaminobenzidine (DAB).

\section{mRNA stability assay}

We used $5 \mathrm{mg} / \mathrm{mL}$ Actinomycin D (Act D; Sigma Aldrich (MO, USA) to test the mRNA stability in C42B and DU145 cell lines as per the manufacturer's protocol. At different time points, cells were harvested. Subsequently, RNA from each cell sample was extracted and RT-qPCR was performed.

\section{In vivo assay}

For the proliferation assays, C4-2B cells of KIF3C knockdown, negative control $\left(1 \times 10^{6} / 200 \mu \mathrm{l}\right)$ were subcutaneously injected into BALB/c nude mice. The tumors were dissected and weighed (4-6 weeks old, male). The animal experiments were approved by the Animal Care Committee of The First Affiliated Hospital of Guizhou medical University. The animal experiments were approved by the Animal Care Committee of The First Affiliated Hospital of Guizhou medical University (approval no. 2006369).

\section{Statistical analysis}

The experimental data was repeated thrice and expressed as mean $\pm \mathrm{SD}$, and all the data were processed by Prism7.0 analysis. A comparison between the groups was performed using Student's t-test, ANOVA and Person analysis. P-value of less than 0.05 indicated a statistically significant difference.

\section{Data availability}

Data supporting the findings of this study are available from the corresponding author upon request.

\section{AUTHOR CONTRIBUTIONS}

HG and JQ designed the study, performed experimental data collection, performed data analysis. FC and QL assisted in experimental design, data review, and manuscript review. JQ supervised study experimental design, assisted with data review and interpretation, and assisted with manuscript writing and review.

\section{CONFLICTS OF INTEREST}

The authors declare that they have no conflicts of interest. 


\section{FUNDING}

The author(s) received no financial support for the research, authorship, and/or publication of this article.

\section{REFERENCES}

1. Siegel RL, Miller KD, Jemal A. Cancer statistics, 2018. CA Cancer J Clin. 2018; 68:7-30. https://doi.org/10.3322/caac.21442 PMID:29313949

2. Pronzato $\mathrm{P}$, Rondini M. Hormonotherapy of advanced prostate cancer. Ann Oncol. 2005 (Suppl 4); 16:iv80-84. https://doi.org/10.1093/annonc/mdi913 PMID:15923436

3. Harris WP, Mostaghel EA, Nelson PS, Montgomery B. Androgen deprivation therapy: progress in understanding mechanisms of resistance and optimizing androgen depletion. Nat Clin Pract Urol. 2009; 6:76-85. https://doi.org/10.1038/ncpuro1296 PMID:19198621

4. Vale RD, Reese TS, Sheetz MP. Identification of a novel force-generating protein, kinesin, involved in microtubule-based motility. Cell. 1985; 42:39-50. https://doi.org/10.1016/s0092-8674(85)80099-4 PMID: $\underline{3926325}$

5. Hirokawa N, Noda Y. Intracellular transport and kinesin superfamily proteins, KIFs: structure, function, and dynamics. Physiol Rev. 2008; 88:1089-118. https://doi.org/10.1152/physrev.00023.2007 PMID:18626067

6. Hirokawa N, Takemura R. Molecular motors and mechanisms of directional transport in neurons. Nat Rev Neurosci. 2005; 6:201-14. https://doi.org/10.1038/nrn1624 PMID:15711600

7. Navone F, Consalez GG, Sardella M, Caspani E, Pozzoli O, Frassoni C, Morlacchi E, Sitia R, Sprocati T, Cabibbo A. Expression of $\mathrm{KIF} 3 \mathrm{C}$ kinesin during neural development and in vitro neuronal differentiation. J Neurochem. 2001; 77:741-53.

https://doi.org/10.1046/i.1471-4159.2001.00277.x PMID:11331403

8. Takeda S, Yamazaki H, Seog DH, Kanai Y, Terada S, Hirokawa N. Kinesin superfamily protein 3 (KIF3) motor transports fodrin-associating vesicles important for neurite building. J Cell Biol. 2000; 148:1255-65.

https://doi.org/10.1083/icb.148.6.1255

PMID:10725338

9. Haruki $T$, Maeta $Y$, Nakamura $S$, Sawata $T$, Shimizu $T$, Kishi K, Miyasaka S, Maeta H, Morimoto K, Taniguchi I. Advanced cancer with situs inversus totalis associated with KIF3 complex deficiency: report of two cases. Surg Today. 2010; 40:162-66. https://doi.org/10.1007/s00595-009-4005-x PMID:20107958

10. Phang HQ, Hoon JL, Lai SK, Zeng $Y$, Chiam KH, Li HY, Koh CG. POPX2 phosphatase regulates the KIF3 kinesin motor complex. J Cell Sci. 2014; 127:727-39. https://doi.org/10.1242/ics.126482 PMID:24338362

11. Jiang L, Wei $Y$, Ronquillo CC, Marc RE, Yoder BK, Frederick JM, Baehr W. Heterotrimeric kinesin-2 (KIF3) mediates transition zone and axoneme formation of mouse photoreceptors. J Biol Chem. 2015; 290:12765-78.

https://doi.org/10.1074/ibc.M115.638437 PMID:25825494

12. Yang Z, Roberts EA, Goldstein LS. Functional analysis of mouse kinesin motor Kif3C. Mol Cell Biol. 2001; 21:5306-11.

https://doi.org/10.1128/MCB.21.16.5306-5311.2001 PMID:11463814

13. Gao Y, Zheng H, Li L, Zhou C, Chen X, Zhou X, Cao Y. KIF3C Promotes Proliferation, Migration, and Invasion of Glioma Cells by Activating the PI3K/AKT Pathway and Inducing EMT. Biomed Res Int. 2020; 2020:6349312. https://doi.org/10.1155/2020/6349312 PMID:33150178

14. Wang C, Wang C, Wei Z, Li Y, Wang W, Li X, Zhao J, Zhou X, Qu X, Xiang F. Suppression of motor protein $\mathrm{KIF} 3 \mathrm{C}$ expression inhibits tumor growth and metastasis in breast cancer by inhibiting TGF- $\beta$ signaling. Cancer Lett. 2015; 368:105-14. https://doi.org/10.1016/j.canlet.2015.07.037 PMID:26272184

15. Yang $Y$, Hsu PJ, Chen YS, Yang YG. Dynamic transcriptomic $\mathrm{m}^{6} \mathrm{~A}$ decoration: writers, erasers, readers and functions in RNA metabolism. Cell Res. 2018; 28:616-24.

https://doi.org/10.1038/s41422-018-0040-8 PMID:29789545

16. Roundtree IA, Evans ME, Pan T, He C. Dynamic RNA Modifications in Gene Expression Regulation. Cell. 2017; 169:1187-200.

https://doi.org/10.1016/i.cell.2017.05.045

PMID:28622506

17. Wang $S$, Chai $P$, Jia $R$, Jia R. Novel insights on $m^{6} A$ RNA methylation in tumorigenesis: a double-edged sword. Mol Cancer. 2018; 17:101.

https://doi.org/10.1186/s12943-018-0847-4

PMID:30031372

18. Wu X, Sang L, Gong Y. N6-methyladenine RNA modification and cancers. Am J Cancer Res. 2018; 8:1957-66.

PMID: $\underline{30416848}$ 
19. He H, Wu W, Sun Z, Chai L. MiR-4429 prevented gastric cancer progression through targeting METTL3 to inhibit $\mathrm{m}^{6} \mathrm{~A}$-caused stabilization of SEC62. Biochem Biophys Res Commun. 2019; 517:581-87. https://doi.org/10.1016/j.bbrc.2019.07.058 PMID:31395342

20. Cheng $M$, Sheng L, Gao $Q$, Xiong $Q$, Zhang $H$, Wu $M$, Liang $Y$, Zhu F, Zhang $Y$, Zhang $X$, Yuan $Q$, Li Y. The $\mathrm{m}^{6} \mathrm{~A}$ methyltransferase METTL3 promotes bladder cancer progression via AFF4/NF-kB/MYC signaling network. Oncogene. 2019; 38:3667-80.

https://doi.org/10.1038/s41388-019-0683-z PMID:30659266

21. Xia T, Wu X, Cao M, Zhang P, Shi G, Zhang J, Lu Z, Wu P, Cai $B$, Miao $Y$, Jiang $K$. The RNA m6A methyltransferase METTL3 promotes pancreatic cancer cell proliferation and invasion. Pathol Res Pract. 2019; 215:152666.

https://doi.org/10.1016/j.prp.2019.152666 PMID:31606241

22. Li F, Mahato RI. MicroRNAs and drug resistance in prostate cancers. Mol Pharm. 2014; 11:2539-52. https://doi.org/10.1021/mp500099g PMID:24742219

23. Chen $X Y$, Zhang J, Zhu JS. The role of $m^{6} A$ RNA methylation in human cancer. Mol Cancer. 2019; 18:103.

https://doi.org/10.1186/s12943-019-1033-z PMID:31142332

24. Huang $H$, Weng $H$, Sun $W$, Qin $X$, Shi $H$, Wu $H$, Zhao BS, Mesquita A, Liu C, Yuan CL, Hu YC, Hüttelmaier $S$, Skibbe JR, et al. Recognition of RNA N $\mathrm{N}^{6}$ methyladenosine by IGF2BP proteins enhances mRNA stability and translation. Nat Cell Biol. 2018; 20:285-95. https://doi.org/10.1038/s41556-018-0045-z PMID:29476152

25. Mishra S, Yadav T, Rani V. Exploring miRNA based approaches in cancer diagnostics and therapeutics. Crit Rev Oncol Hematol. 2016; 98:12-23. https://doi.org/10.1016/j.critrevonc.2015.10.003 PMID:26481951

26. Nelson WG, De Marzo AM, Isaacs WB. Prostate cancer. N Engl J Med. 2003; 349:366-81.
https://doi.org/10.1056/NEJMra021562 PMID:12878745

27. Rath O, Kozielski F. Kinesins and cancer. Nat Rev Cancer. 2012; 12:527-39. https://doi.org/10.1038/nrc3310 PMID:22825217

28. Son HJ, Choi EJ, Yoo NJ, Lee SH. Somatic frameshift mutations of cancer-related genes KIF3C and BARD1 in colorectal cancers. Pathol Res Pract. 2019; 215:152579. https://doi.org/10.1016/j.prp.2019.152579 PMID:31400927

29. Frye M, Harada BT, Behm M, He C. RNA modifications modulate gene expression during development. Science. 2018; 361:1346-49.

https://doi.org/10.1126/science.aau1646 PMID:30262497

30. Zhao BS, Roundtree IA, He C. Post-transcriptional gene regulation by mRNA modifications. Nat Rev Mol Cell Biol. 2017; 18:31-42. https://doi.org/10.1038/nrm.2016.132 PMID:27808276

31. Meyer KD, Jaffrey SR. Rethinking $\mathrm{m}^{6} \mathrm{~A}$ Readers, Writers, and Erasers. Annu Rev Cell Dev Biol. 2017; 33:319-42. https://doi.org/10.1146/annurev-cellbio-100616060758 PMID:28759256

32. Lin S, Choe J, Du P, Triboulet R, Gregory RI. The $m(6) A$ Methyltransferase METTL3 Promotes Translation in Human Cancer Cells. Mol Cell. 2016; 62:335-45. https://doi.org/10.1016/i.molcel.2016.03.021 PMID:27117702

33. Barbieri I, Tzelepis K, Pandolfini L, Shi J, MillánZambrano G, Robson SC, Aspris D, Migliori V, Bannister AJ, Han N, De Braekeleer E, Ponstingl H, Hendrick A, et al. Promoter-bound METTL3 maintains myeloid leukaemia by $\mathrm{m}^{6} \mathrm{~A}$-dependent translation control. Nature. 2017; 552:126-31. https://doi.org/10.1038/nature24678 PMID:29186125

34. Rupaimoole R, Slack FJ. MicroRNA therapeutics: towards a new era for the management of cancer and other diseases. Nat Rev Drug Discov. 2017; 16:203-22. https://doi.org/10.1038/nrd.2016.246 PMID:28209991 


\section{SUPPLEMENTARY MATERIALS}

\section{Supplementary Figures}

A

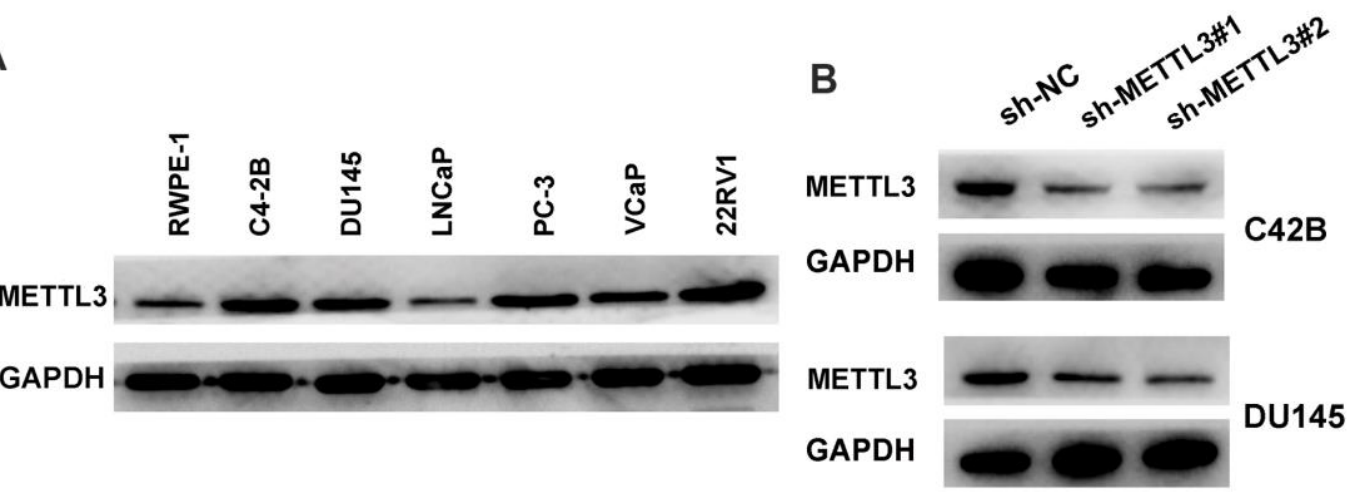

Supplementary Figure 1. (A) Western blotting showing METTL3 expression in PCa cell lines. (B) Western blotting showing METTL3 knockdown expression in C4-2B and DU145 cells.

\begin{tabular}{|c|c|c|c|c|c|c|c|}
\hline Show $10 \vee$ entries & & & & & & & \\
\hline SitelD & $\uparrow$ & StackedRegion & 11 & BindTranscriptNum & $\uparrow \downarrow$ & ClipExpNum & †1 \\
\hline IGF2BP1:CH000109563 & $\uparrow$ & chr2:26149868-26149873[-] & 1 & 3 & $\uparrow$ & 2 & $\uparrow$ \\
\hline IGF2BP1:CH000109564 & & chr2:26150278-26150319[-] & & 3 & & 3 & \\
\hline IGF2BP1:CH000109565 & & chr2:26150936-26150957[-] & & 3 & & 3 & \\
\hline IGF2BP1:CH000109566 & & chr2:26151028-26151033[-] & & 3 & & 1 & \\
\hline IGF2BP1:CH000109567 & & chr2:26151159-26151164[-] & & 3 & & 3 & \\
\hline IGF2BP1:CH000109568 & & chr2:26151327-26151328[-] & & 3 & & 3 & \\
\hline IGF2BP1:CH000109569 & & chr2:26151378-26151433[-] & & 3 & & 1 & \\
\hline
\end{tabular}

Supplementary Figure 2. Bioinformatic analysis the KIF3C RNA binding protein IGF2BP1. 